\title{
Study on Motion Management of Helical Tomotherapy Specially Lung Cancer
}

\author{
Md Tofiz Uddin* \\ Department of Medical Physics, Harbin Medical University, China
}

Submission: March 21, 2019; Published: April 24, 2019

"Correspondence Author: Md Tofiz Uddin, Department of Medical Physics, 150th Affiliated Cancer Hospital, Harbin Medical University, Harbin, China

\begin{abstract}
Purpose: Tomotherapy consists of helical radiotherapy using a computed tomography (CT)-like gantry and a rotating radiation beam that passes through the target area of interest; this modality has been used in the management of primary central nervous system tumors and viscerabased malignancies. It provides unprecedented accuracy in beam delivery allowing for an increase in tumour dose, thereby increasing the likelihood of cancer cure while at the same time reducing treatment complications in healthy tissues. The main purpose of Study is to Investigate Tumor motion limitation for effective radiotherapy of Lung Cancer specifically for Helical Tomotherapy in Harbin Medical University Affiliated Cancer Hospital. Simultaneous and continuous movements of tomotherapy subsystems (gantry, couch, and binary multi-leaf collimator) can lead to inaccurate dose delivery, when combined with tumor motion.
\end{abstract}

Methods: In this present study, we have investigated the influence of tumour motion and strategies to reduce the resulting dose differences for helical tomotherapy, through computer simulations and film measurements performed in a dynamic body phantom. Three distinctively different types of dose discrepancies have been isolated: dose rounding, dose rippling, and the intensity-modulated radiation therapy (IMRT) asynchronization effect. Each effect was shown to be affected by different combinations of tumour motion and treatment parameters. In clinical practice using a conventional fractionation scheme, the dose rounding effect remains the major concern, which can be compensated by assigning a larger treatment margin around the tumour volume. For hypofractionation schemes, the IMRT asynchronization effect can become an additional concern by introducing dose discrepancies inside the target volume, necessitating the use of a motion management technique.

Results: Three different types of dissimilarities arising from the presence longitudinal tumour motion during helical tomotherapy were identified. Their characteristics were investigated through both computer simulation modeling and experimental verification using a motion phantom with film. The characteristics of the three different motion-induced dose discrepancies were illustrated, and the dosimetric significance of each effect was described.

Conclusion: Investigated measured data within $+0.5 \%$ and $+1.5 \%$ inside the PTV region for the non-IMRT and IMRT tomotherapy deliveries, respectively. Various potential solutions to minimize the effect of target motion were also discussed, and each proposed method needs to be investigated for its effectiveness in suppressing the three dose discrepancies described here. The computer simulation model could also prove useful during the patient selection process for the different approaches of minimizing dose artifacts

Keywords: Motion Management; Helical Tomotherapy; Lung Cancer; Respiratory Gating

\section{Introduction}

Lung cancer has been the leading cancer diagnosed and cause of cancer death for many years in China, with a rapidly increasing trend during the past several decades [1,2] The incidence rate of lung cancer in China is relatively high, but is increasing at a more rapid rate than in Western countries. Many risk factors, such as cigarette smoking and air pollution, have been proven as risk factors of the disease [3-6]. With a large smoking population, the growth of lung cancer incidence in China will continue to rise. This article provides an up-to-date description of the epidemiology of lung cancer in China, which will provide an evidence base for future interventions to improve health in China.
Lung cancer has been the most common cancer in the world for several decades. There are estimated to be 1.8 million new cases in 2012 (12.9\% of the total), 58\% of which occurred in less developed regions. The disease remains the most common cancer in men worldwide (1.2 million, $16.7 \%$ of the total), with the highest estimated age-standardised incidence rates in Eastern Asia (50.4 per 100000 ). Lung cancer is the most common cause of cancer death worldwide, estimated to be responsible for nearly one in five deaths (1.59 million deaths, $19.4 \%$ of the total). Because of its high fatality (the overall ratio of mortality to incidence is 0.87 ) and the relative lack of variability in survival in different world regions, the geographical patterns in mortality closely follow those 


\section{Cancer Therapy \& Oncology International Journal}

of incidence. As the most populous country in the world, China contains $19 \%$ of the word population with $21.75 \%$ of all newly diagnosed cancer cases and $26.90 \%$ of deaths, including $35.78 \%$ of all newly diagnosed lung cancer cases and $37.56 \%$ of lung cancer deaths worldwide [7].

Traditional linear accelerators are currently limited to serial tomotherapy due to the limited rotation possible and the inability to move the couch smoothly and automatically during radiation delivery [8-13]. Furthermore, serial tomotherapy is unable to image the patient in treatment position and, therefore, unable to assure the accurate placement of the high dose volume with respect to the malignant region. The tomotherapy unit under development at Madison, WI (TomoTherapy Inc.) seeks to remove these limitations. As can be seen in (Figure 1), the helical tomotherapy machine is a combination of a helical CT scanner and a linear accelerator. It uses the slip ring technology of diagnostic CT scanners and, therefore, the unit is capable of continuous rotation around the patient while the couch is moving into the gantry, thus providing smooth helical delivery as shown in (Figure 1) $2^{\text {nd }}$ part. Mounted on the rotating gantry and attached to the slip ring is a compact ( $\sim 40 \mathrm{~cm}$ long) $6 \mathrm{MeV}$ S-band ( $3 \mathrm{GHz}$ ) linear accelerator generating a $6 \mathrm{MV}$ photon beam [14]. The beam from the accelerator is collimated by a multileaf collimator (Figure 2c) consisting of 64 leaves each of which project a shadow of $6.25 \mathrm{~mm}$ at the patient generating a total fan beam width of $40 \mathrm{~cm}$. By using a separate collimation ("jaws") system above the multileaf collimators, the "slice thickness" can range between 0.5 to $5 \mathrm{~cm}$. Since it is a specially designed machine for helical, fan beam delivery, the multileaf collimation system is specifically designed to minimize leaf transmission and interleaf leakage - important considerations for narrow beam, multislice delivery procedures [15-17].

The on-board megavoltage CT detection system is from a conventional, commercial diagnostic scanner using xenon detectors. Similarly, the patient couch is also from a commercial scanner. The CT detection system can be used for: (1) patient set-up verification and repositioning, if necessary, (2) verification of leaf positions during treatment, and (3) reconstruction of the actual dose delivered to the patient with the possibility of making corrections in subsequent fractions. Although megavoltage CT images generally have inferior tissue contrast compared with kilovoltage CT, research to date demonstrates that imaging using megavoltage photons may be adequate for set-up verification, delivery verification and dose reconstruction purposes. To improve image quality and reduce the dose to the patient, when used for imaging purposes, the linear accelerator is slightly detuned leading to a maximum electron energy at the target of 3.5 rather than $6 \mathrm{MeV}$. While the schematic in (Figure 1) shows both megavoltage and kilovoltage imaging capabilities, the first helical tomotherapy units will not Incorporate the kilovoltage capabilities. These will only be added if a clinical need is demonstrated [18-21].



The research work done in this thesis was focused on the following objectives: The research work was to investigate the different types of dose dissimilarities resulting from tumour motion during HT(Helical Tomotherapy), and investigate the characteristics of each dose discrepancy individually, in relations to different treatment and tumour motion parameters, through computer simulations and experimental measurements using film dosimetry, investigate the dosimetric characteristics of the loose helical tomotherapy delivery with breath-holding, simulated on a linear accelerator (prior to the arrival of the helical tomotherapy unit at LRCP), through treatment planning and experimental measurements using film dosimetry and investigate the feasibility of employing multi-pass respiratory gating technique for helical tomotherapy, through computer simulations and experimental measurements using film dosimetry on the helical tomotherapy machine [21-24]. 


\section{Cancer Therapy \& Oncology International Journal}

a) Room coordinate system ( $Y$ )

$40 \mathrm{~cm}$



couch b) Tumor coordinate system (y)

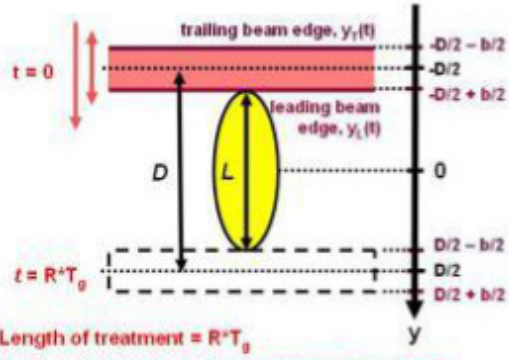

Total couch travel during treatment $=D=b^{*} p^{*} R$

Length of target $=L=D-b=b^{\star} p^{\top} R-b$

where $R$ is the number of gantry rotations during

treatment, $T_{g}$ is the gantry rotation period, $b$ is the

fan beam thickness. and $\mathrm{p}$ is the pitch factor.

Figure 2: Coordinate systems for a moving target: a) In the room coordinate system, the tumour position (Y) with respect to the stationary fan beam is governed by the linear motion of the treatment couch, combined with the periodic motion of the tumour volume itself, as summarized in Equation 2, In the tumor coordinate system (y), the target volume remains stationary, while the "moving" treatment field assumes the roles of both couch motion and tumor motion. The two fan beam edges (trailing beam edge, yT ( $\mathrm{t}$ ) and leading beam edge, yL $(\mathrm{t})$ move along the longitudinal direction $(+\mathrm{y})$ with respect to the center of the tumor volume according to Equation 3 .

\section{Methods and Materials}

The treatment parameters associated with helical tomotherapy include pitch factor ( $\mathrm{p}$ ), fan beam thickness (b), gantry rotation period $\left(\mathrm{T}_{\mathrm{g}}\right)$, and number of gantry rotations $(\mathrm{R})$. Pitch factor $(\mathrm{p})$ is defined as the progression distance of the longitudinal couch motion per gantry rotation in units of fan beam thickness (b). Gantry rotation period $\left(\mathrm{T}_{\mathrm{g}}\right)$ is the time required to complete one full gantry rotation; the current helical tomotherapy unit allows for selectable periods $\left(\mathrm{T}_{\mathrm{g}}\right)$ between 15 and $60 \mathrm{~s}$, with a gantry rotation period of 15 to $20 \mathrm{~s}$ being used clinically for the helical tomotherapy of lung cancer [25]. Number of gantry rotations (R) is the number of gantry rotations required to treat the target volume during the treatment. The parameters related to tumour motion are amplitude $(A)$ and period $\left(T_{r}\right)$. Amplitude $(A)$ is defined as the onehalf of the peak-to-peak amplitude (2A) of tumour motion along the longitudinal direction $( \pm \mathrm{Y})$, while tumour motion period $\left(\mathrm{T}_{\mathrm{r}}\right)$ is the time required to complete one full cycle of tumour motion.

The current helical tomotherapy unit (HI-ART II) allows the user to enter a leaf opening sinogram directly at the treatment console for a radiation delivery. In order for the leaf opening sinogram to be accepted at the treatment console, the leaf opening sinogram must contain values of relative leaf opening time. Relative leaf opening time $\left(\mathrm{T}_{0}\right)$ is defined as the ratio of the actual leaf opening time $\left(\mathrm{T}_{\mathrm{a}}\right.$ ) for a beam projection to the maximum leaf opening time per beam projection $\left(\mathrm{T}_{\mathrm{m}}\right)$ :

$$
T_{0}=\frac{T_{a}}{T m}, \mathrm{~T}_{m}=\frac{T_{g}}{n}
$$

where $\mathrm{T}_{0}$ is the relative leaf opening time for a beam projection, $\mathrm{T}_{\mathrm{a}}$ is the actual leaf opening time for the beam projection, $\mathrm{T}_{\mathrm{m}}$ is the maximum leaf opening time per beam projection, $\mathrm{T}_{\mathrm{g}}$ is the gantry rotation period, and $\mathrm{n}$ is the number of beam projections per gantry rotation $(n=51)$. For the non-IMRT plan, the planning target volume (PTV) was assumed to be a $5.5 \mathrm{~cm}$-long cylinder centered along the axis of gantry rotation inside the body phantom (Modus Medical Devices Inc., London, Canada) shown in (Figure 1a), which mimicked a human torso. The four central leaves were assigned with the maximum leaf opening time $\left(\mathrm{T}_{0}=\right.$ $\mathrm{T}_{\mathrm{m}}=1.00$ ) for all beam projections, while the rest of the leaves remained fully closed $\left(\mathrm{T}_{0}=0.00\right)$ during the entire treatment, using a fan beam thickness (b) of $2.5 \mathrm{~cm}$. A fixed value of leaf opening time was assigned for each individual leaf, so that a uniform fluence was delivered only along the central axis of the PTV. Using a pitch factor of $0.8(\mathrm{p}=0.8)$, four full gantry rotations $(\mathrm{R}=4)$ were required to treat the PTV, and a gantry rotation period of $16 \mathrm{~s}\left(\mathrm{~T}_{\mathrm{g}}\right.$ $=16 \mathrm{~s}$ ) was selected. A pitch factor of 0.8 was selected rather than the typically used pitch factor of less than 0.3 for lung cancer tomotherapy [25].

An IMRT helical tomotherapy plan was generated using the CT images of a body phantom $\left(\rho=1.18 \mathrm{~g} / \mathrm{cm}^{3}\right.$, length $=12 \mathrm{~cm}$, width $=30 \mathrm{~cm}$, height $=20 \mathrm{~cm}$ ) in (Figure 1a). On the CT images of the body phantom (Figure 1b), a virtual $3.6 \mathrm{~cm}$-long cylindrical organ at risk (OAR) was delineated to be wrapped around by a $6.6 \mathrm{~cm}$-long C-shaped planning target volume (PTV). Contouring of the PTV, the OAR, and the phantom outline was performed in a commercial treatment planning system (Pinnacle 8.0d, Philips Medical Systems). The CT images and contours were then exported to the tomotherapy treatment planning system (TomoTherapy version 2.2.2, TomoTherapy Inc., Madison, WI), where a dose of 2.0 Gy was prescribed to $95 \%$ of the PTV with the maximum OAR dose being limited to $0.8 \mathrm{~Gy}$. A pitch factor $(\rho)$ of 0.287 (i.e. $0.86 / 3$ ) was selected to minimize the "thread artifact" [26], and a fan beam thickness of $2.5 \mathrm{~cm}$ was chosen. During the tomotherapy plan optimization process, the values of leaf opening time needed to optimize the plan were calculated for each of the $64 \mathrm{~b}$-MLC leaves as a function of beam projection, yielding the planned dose distribution shown in (Figure 1c).

At the end of the plan optimization, the generated leaf opening sinogram contained sums of leaf opening time for all fractions of a treatment (i.e. actual leaf opening time per fraction * number of fractions) over 819 beam projections ( $\mathrm{R} \sim 16)$, with the matrix 
rows and columns representing beam projections and leaf number, respectively. The leaf opening sinogram was then truncated to shorten the length of the PTV from $6.6 \mathrm{~cm}$ to $3.24 \mathrm{~cm}$, so that the resulting PTV region fell well within the length of the body phantom with the motion amplitudes used in this study. This was done by eliminating the first 252 and the last 159 beam projections, yielding a plan with 408 beam projections $(\mathrm{R}=8)$ and a plan dose of 1.75 Gy instead of 2.0 Gy. This was deemed acceptable, as the objective of this study was not to reproduce a plan, but to investigate how motion affects a given plan 1 . In order to make the modified leaf opening sinogram to be accepted at the treatment console, each value of leaf opening time was converted to relative leaf opening time, and the entire leaf opening sinogram matrix was then transposed (i.e. indexed by leaf number, beam projection) [27].

The tumour coordinate system (Figure $2 \mathrm{~b}$ ) was adopted for the computer simulation model in this study, instead of the room coordinate system (Figure 2a) used by Kissick et al. [20]. In the room coordinate system (Figure $2 \mathrm{a}$ ), the tumour position, $\mathrm{Y}(\mathrm{t})$ is determined by the linear motion of the treatment couch combined with the periodic motion of the tumour volume itself:

$$
Y(t)=Y_{\text {couch }}(t)+Y_{\text {tumour }}(t)=\frac{p \cdot b}{T_{g}} t+A \sin \frac{2 \pi}{T_{r}} t
$$

where ${ }_{\text {couch }^{(t)}}$ is the position of the treatment couch as a function of time, $Y_{\text {couch }}(t)$ is the position of the tumour volume on the treatment couch as a function of time, $p$ is the pitch factor, $b$ is the fan beam thickness, $\mathrm{Tg}$ is the gantry rotation period, $\mathrm{Tr}$ is the period of tumor motion, and $\mathrm{A}$ is the amplitude of tumour motion along the longitudinal $( \pm \mathrm{Y})$ direction.

In the tumour coordinate system (y) shown in (Figure $2 \mathrm{~b}$ ), the tumour volume remains stationary, while the "moving" treatment field assumes the roles of both couch motion and tumour motion. The two fan beam edges, leading beam edge $y_{L}(t)$ and trailing beam edge $y_{T}(t)$ move along the longitudinal direction $( \pm y)$ with respect to the center of the tumor volume according to the following equations:

$$
\begin{aligned}
& Y_{T}(t)=\frac{p \cdot b}{T_{g}} t+A \sin \frac{2 \pi}{T_{r}} t-\frac{D}{2}-\frac{b}{2}=\frac{p \cdot b}{T_{g}} t+A \sin \frac{2 \pi}{T_{r}} t-\frac{p \cdot b \cdot R}{2}-\frac{b}{2} \\
& Y_{L}(t)=\frac{p \cdot b}{T_{g}} t+A \sin \frac{2 \pi}{T_{r}} t-\frac{D}{2}+\frac{b}{2}=\frac{p \cdot b}{T_{g}} t+A \sin \frac{2 \pi}{T_{r}} t-\frac{p \cdot b \cdot R}{2}+\frac{b}{2}
\end{aligned}
$$

where $\mathrm{y}_{\mathrm{T}} \mathrm{T}(\mathrm{t})$ and $\mathrm{y}_{\mathrm{L}}(\mathrm{t})$ are the positions of the trailing and leading beam edges as functions of time, respectively, $D$ is the total couch travel during the treatment, and $\mathrm{R}$ is the number of gantry rotations used during the treatment (Figure 2).

The dose received by each longitudinal point (y) along the axis of gantry rotation was determined by first calculating the radiation exposure time. In (Figure 3), a set of solid lines observed at each longitudinal position represents the irradiation time ("ON") of the corresponding point, while the dotted lines indicate the time spent outside the longitudinal beam edges ("OUT"). Thus, the radiation exposure time $(\Delta \mathrm{t})$ was calculated by summing up the temporal lengths of all solid's lines:

$$
\Delta t(y)=\sum_{i=1}^{m} \Delta t_{i}(y)
$$

where $\Delta t(y)$ is the radiation exposure time at a given longitudinal position ( $y$ ), $m$ is the number of solid lines at each longitudinal position $(y)$, and $t_{i}$ is temporal length of the $i^{\text {th }}$ solid line.

For the non-IMRT helical tomotherapy cases shown in (Figures $3 \mathrm{a} \& \mathrm{~b}$ ), the irradiated time ("ON") was equal to the time spent inside the two fan beam edges ("IN"), since the radiation beam was fully on during the entire treatment by assigning the maximum leaf opening time for all beam projections $(\mathrm{T} 0=1.00)$. For the IMRT helical tomotherapy delivery in (Figure 3c), the irradiated time ("ON") was the temporal overlaps between the time spent inside the beam edges ("IN") and the "Beam ON" windows, making "ON" a subset of "IN". The widths of the "Beam ON" windows were determined by the values of leaf opening time assigned as a function of leaf number and beam projection. The temporal length $\left(\Delta \mathrm{t}_{\mathrm{i}}\right)$ of each solid line ("ON") was calculated numerically by comparing the longitudinal position of the given point to the positions of the beam edges and the "Beam ON" windows in temporal increments of $1 \mathrm{~ms}$. The radiation exposure time $\left(\sum \mathrm{t}_{\mathrm{i}}\right)$ of each point was then determined by summing up the lengths of all the irradiated time segments $\left(\Delta \mathrm{t}_{\mathrm{i}}^{\prime} \mathrm{s}\right)$. The radiation exposure time calculation was repeated in spatial increments of $0.01 \mathrm{~cm}$ to produce a radiation exposure time versus position profile.

The resulting longitudinal exposure time versus position profile was converted directly into a longitudinal fluence profile by assuming a constant fluence rate. The fluence profile, $\Phi(\mathrm{y})$ was then converted to a longitudinal dose profile, $\mathrm{D}(\mathrm{y})$ via one-dimensional convolution using an empirically determined dose kernel, $\mathrm{K}(\mathrm{y})$ :

$$
D(y)=K(y) \otimes \Phi(y)
$$

where $\mathrm{D}(\mathrm{y})$ is the dose profile, $\mathrm{K}(\mathrm{y})$ is the dose kernel, and $\Phi(\mathrm{y})$ is the normalized fluence profile. The normalized fluence profile $\Phi(y)$ was assumed to be rectangular in shape along the direction. The dose kernel, $\mathrm{K}(\mathrm{y})$ was then derived using a dose profile along the axis of gantry rotation, acquired experimentally using Kodak EDR2 film. A piece of film was placed inside the stationary film insert within the body phantom (Figure 1a) and irradiated for one full gantry rotation $\left(\mathrm{T}_{\mathrm{g}}=15 \mathrm{~s}\right)$ with the treatment couch remaining stationary during the radiation delivery. The dose kernel was a set of exponential functions resembling the primary beam penumbra equations defined by Cunnigham [27] and used by a commercial treatment planning system (Theraplan Plus v.3.0, 1998) [22]. The dose profile calculated with the dose kernel, $\mathrm{K}(\mathrm{y})$ was fitted manually to the measured dose profile by continuously varying the coefficient values of the different parameters in the dose kernel, $\mathrm{K}(\mathrm{y})$. The fitting process was repeated until the root- mean-square (RMS) of the absolute differences between the entire calculated and measured dose profiles was less than $1.2 \%$.

For each film experiment, a calibration procedure was carried out with a $6 \mathrm{MV}$ helical tomotherapy X-ray beam, using a cylindrical solid water "Cheese" phantom (TomoTherapy Inc., Madison, 


\section{Cancer Therapy \& Oncology International Journal}

WI, length $=18 \mathrm{~cm}$, diameter $=30 \mathrm{~cm}$ ). A 10" x 12" EDR2 film was placed inside the phantom, and the couch height was adjusted to position the film on the isocentrical coronal plane. The calibration procedure delivered eight $3 \mathrm{~cm}^{2}$ square fields with known dose of 30.5 cGy, 65.2 cGy, 100.9 cGy, 128.2 cGy, 159.5 cGy, 188.9 cGy, 215.0 cGy, and 244.4 cGy. The calibration film was processed along with a blank film to enable background subtraction. The developed films were then scanned with a Vidar VXR-16 Dosimetry-
Pro scanner (Vidar Systems Corporation, Herndon, VA) and analyzed with the RIT113 V4 software (Radiation Therapy Dosimetry Software, Colorado Springs, CO). A region of interest (ROI) of 2 $\mathrm{cm}^{2}$ was placed inside each of the eight different fields on the calibration film and within the blank film. The average optical density (OD) was then calculated for each ROI to generate an OD versus dose calibration curve.
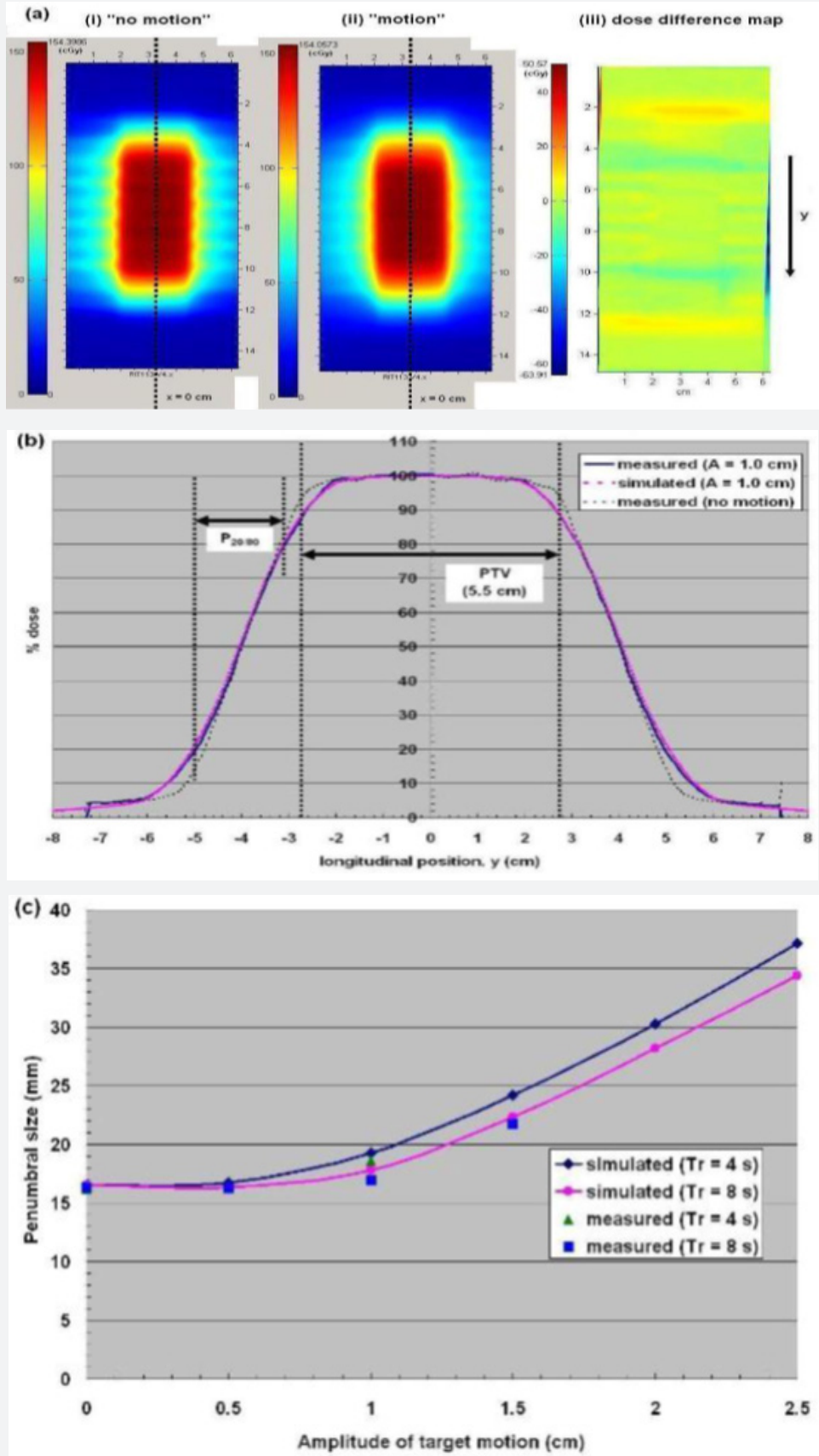

Figure 3: In Dose rounding effect non IMRT helical tomotherapy (a) Measured film dose distributions on the central coronal plane for a non-IMRT helical tomotherapy $(p=8, T g=16 \mathrm{~s}, \mathrm{R}=4)$ of a central target: in the (i) absence and (ii) presence of target motion $(T r=4 \mathrm{~s}$, $\mathrm{A}=1.0 \mathrm{~cm}$, = 5). (i) "no motion" dose distribution, (ii) "motion" dose distribution ( $\operatorname{Tr}=4 \mathrm{~s}, \mathrm{~A}=1.0 \mathrm{~cm}$, =5), and (iii) dose difference map between (i) and (ii). The dotted lines represent the axis of gantry rotation $(x=0 \mathrm{~cm})$. (b) Normalized longitudinal dose profiles along the axis of gantry rotation for a non-IMRT helical tomotherapy $(p=0.8, T g=16 \mathrm{~s}, \mathrm{R}=4)$ of a central target $(\mathrm{Tr}=4 \mathrm{~s}, \mathrm{~A}=1.0 \mathrm{~cm})$. P20/80 is the distance between the $20 \%$ and $80 \%$ dose points, used to quantify penumbral size.(c) Penumbral size vs. amplitude of target motion for two different target motion periods $(\mathrm{Tr}=4 \mathrm{~s}$ and $8 \mathrm{~s})$ for a non-IMRT helical tomotherapy $(p=0.8, T g=16 \mathrm{~s}, \mathrm{R}=4)$ of a central target. 


\section{Cancer Therapy \& Oncology International Journal}

All the film experiments were carried out in the non-clinical mode at the helical tomotherapy unit. An electronic communication was established between the tomotherapy machine and the motion phantom through a cable, such that the film insert motion would commence once the gantry angle reached zero. The initial phase of the film insert motion was set at zero for all non- IMRT and IMRT cases by positioning the film insert in its mid-position before the start of each radiation delivery.
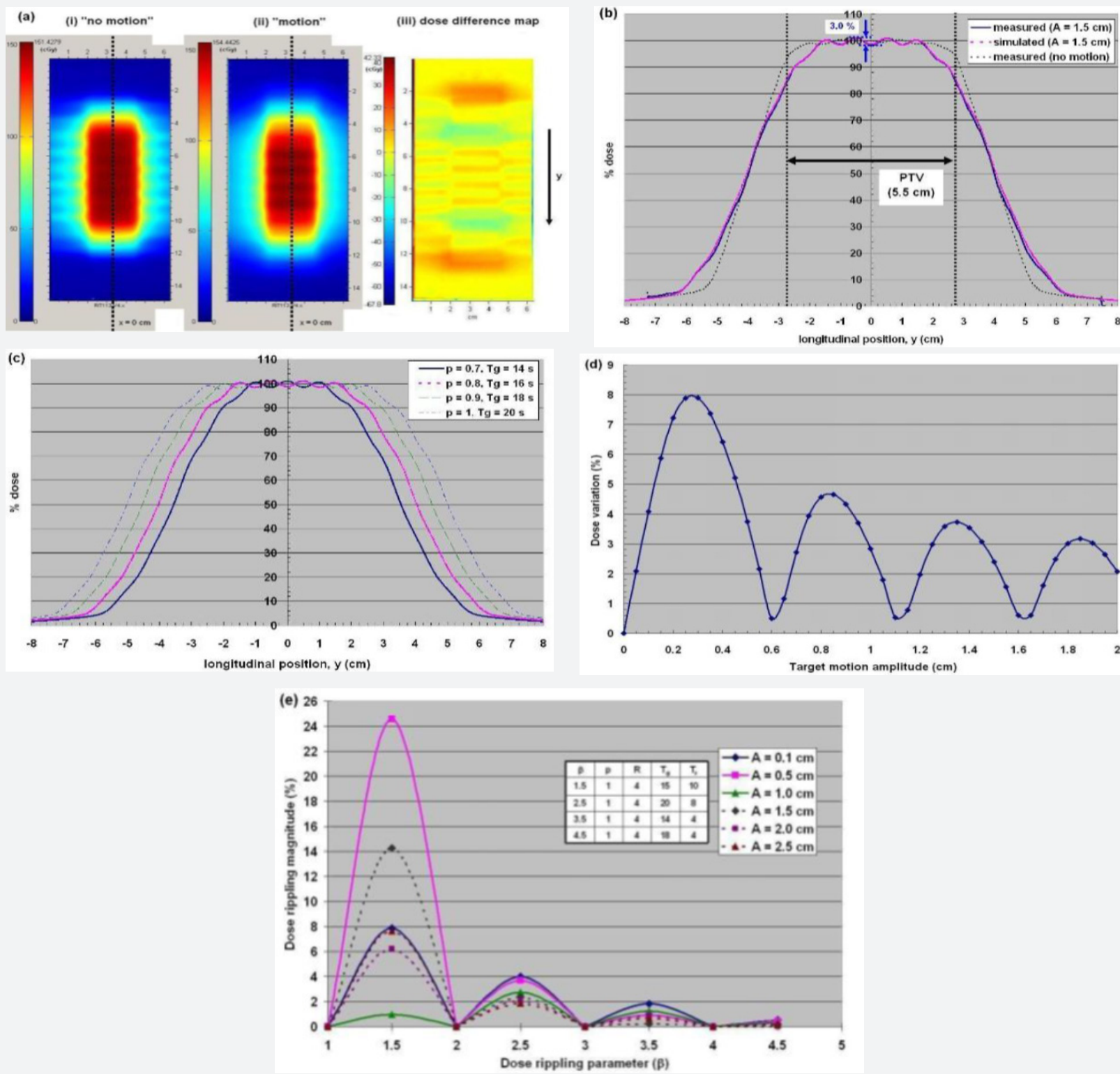

Figure 4: In dose rippling effect non IMRT helical tomotherapy-(a) Measured film dose distributions on the central coronal plane for a nonIMRT helical tomotherapy ( $p=0.8, \mathrm{Tg}=16 \mathrm{~s}, \mathrm{R}=4$ ) of a central target: (i) "no motion" dose distribution, (ii) "motion" dose distribution ( $\mathrm{Tr}$ $=8 \mathrm{~s}, \mathrm{~A}=1.5 \mathrm{~cm}, \quad=2.5$ ), and (iii) dose difference map between (i) and (ii). The dotted lines represent the axis of gantry rotation ( $\mathrm{x}$ $=0 \mathrm{~cm})$. (b) Normalized longitudinal dose profiles along the axis of gantry rotation for a non-IMRT helical tomotherapy $(p=0.8, T g=16 \mathrm{~s}, \mathrm{R}$ $=4$ ) of a central target $(\operatorname{Tr}=8 \mathrm{~s}, \mathrm{~A}=1.5 \mathrm{~cm},=2.5)$. The dose variation was measured as the difference between the lowest dose valley and the highest dose peak found inside the PTV region. (c) Simulated longitudinal dose profiles along the axis of gantry rotation for a non-IMRT helical tomotherapy of a central target $(\operatorname{Tr}=8 \mathrm{~s}, \mathrm{~A}=1.5 \mathrm{~cm},=2.5)$. Four different dose profiles were generated using different combinations of pitch factor $(\mathrm{p})$ and gantry rotation period $(\mathrm{Tg})$, while keeping the value at 2.5. (d) Simulation study: Dose variation vs. target motion amplitude $(\operatorname{Tr}=8 \mathrm{~s})$ for a non-IMRT helical tomotherapy $(p=0.8, T g=16 \mathrm{~s}, \mathrm{R}=4)$ of a central target under the asynchronous interplay condition ( $\neq$ integer $=2.5$ ). (e) Simulation study: Dose rippling magnitude vs. dose rippling parameter () for different amplitudes of target motion for a non-IMRT helical tomotherapy $(p=0.8, T g=16 \mathrm{~s}, \mathrm{R}=4)$ of a central target.

Results and Discussion

For the Non-IMRT Tomotherapy cases dose rounding effect, dose rippling effect and IMRT Helical Tomotherapy Case was illustrated in the measured coronal dose distribution of a non- IMRT helical tomotherapy and IMRT Helical Tomotherapy delivery (Figures 3-5).

Some approaches have been proposed to minimize the effect of tumour motion during helical tomotherapy [1,11-13]. Increas- 


\section{Cancer Therapy \& Oncology International Journal}

ing the PTV margin size along the direction of target motion is the simplest way to compensate for the dose rounding effect, at the expense of increasing the integral dose to the patient. In the penumbral widening due to dose rounding was shown to be proportional to the target motion amplitude (A), thus making the PTV margin size directly dependent upon the target motion amplitude. However, the dose rippling and IMRT leaf opening asynchronization effects cannot be resolved by this approach alone, as the asynchronous interplay conditions remain. The effects of all three dose artifacts could be minimized effectively by immobilizing the target volume only during the "beam ON" time through breath- holding $[11,13]$. However, breath-holding may not be feasible physically for all lung cancer patients, due to their compromised lung functions. In the "motion-incorporated helical tomotherapy", the tumour motion information acquired prior to the treatment is utilized during the plan optimization process $[12,23]$. As a result, the tumour motion is accounted for during the treatment, while the patient is still allowed to breathe normally. This method, however, requires the patient to reproduce the identical breathing pattern used for planning during the treatment, which may not be guaranteed in practice. Failure to reproduce the same breathing pattern can result in significant dose discrepancies between the planned and delivered dose distributions.
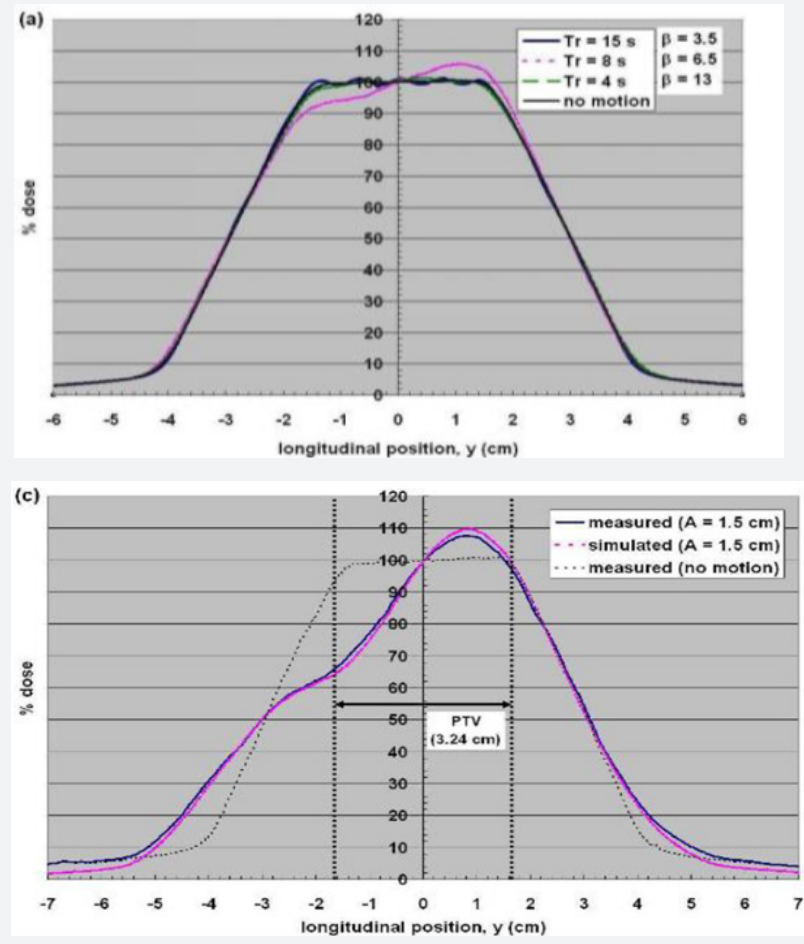
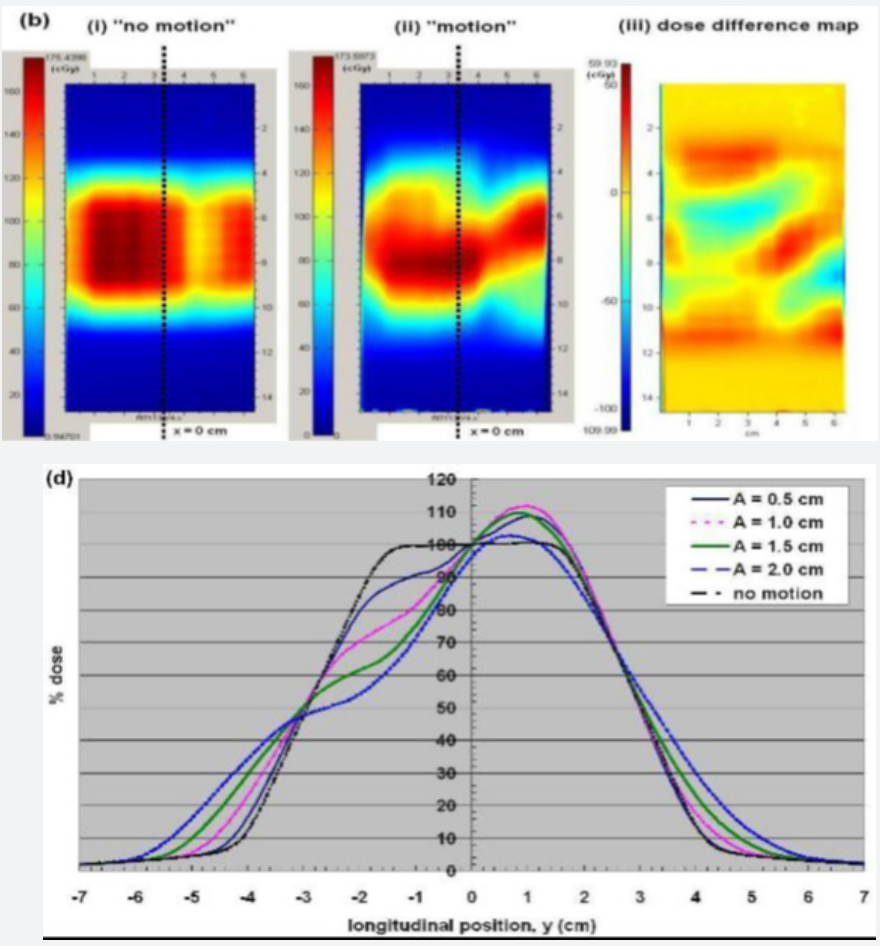

Figure 5: In IMRT helical tomotherapy (a) Simulated longitudinal dose profiles along the axis of gantry rotation for an IMRT helical tomotherapy $(p=0.287, \mathrm{Tg}=15 \mathrm{~s}, \mathrm{R}=8)$ of an off-axis target $(\mathrm{A}=0.3 \mathrm{~cm})$ for different target motion periods (Tr). (b) Measured film dose distributions on the central coronal plane for an IMRT helical tomotherapy ( $p=0.287, \mathrm{Tg}=15 \mathrm{~s}$ ) of an off-axis target: (i) "no motion" dose distribution, (ii) "motion" dose distribution ( $\mathrm{Tr}=8 \mathrm{~s}, \mathrm{~A}=1.5 \mathrm{~cm}$ ), and (iii) dose difference map between (i) and (ii). The dotted lines represent the axis of gantry rotation $(x=0 \mathrm{~cm}$ ). (c) Normalized longitudinal dose profiles along the axis of gantry rotation for an IMRT helical tomotherapy $(p=0.287, \operatorname{Tg}=15 \mathrm{~s}, \mathrm{R}=8)$ of an off-axis target $(\mathrm{Tr}=8 \mathrm{~s}, \mathrm{~A}=1.5 \mathrm{~cm})$. (d) Simulated longitudinal dose profiles along the axis of gantry rotation for an IMRT helical tomotherapy $(p=0.287, T g=15 \mathrm{~s}, \mathrm{R}=8)$ of an off-axis target $(\mathrm{Tr}=8 \mathrm{~s})$ for different target motion amplitudes (A). (e) Simulated longitudinal dose profiles along the axis of gantry rotation for an IMRT helical tomotherapy $(p=0.287, \operatorname{Tg}=$ $15 \mathrm{~s}, \mathrm{R}=8)$ of an off-axis target $(\mathrm{Tr}=8 \mathrm{~s}, \mathrm{~A}=1.5 \mathrm{~cm})$ for 30 fractions.ww

\section{Conclusion}

In this study, three different types of dissimilarities arising from the presence longitudinal tumour motion during helical tomotherapy were identified. Their characteristics were investigated through both computer simulation modeling and experimental verification using a motion phantom with film. The characteristics of the three different motion-induced dose discrepancies were illustrated, and the dosimetric significance of each effect was described. The

computer simulation model was shown to be a valuable tool for predicting the dosimetric impact of target motion during helical tomotherapy, as the calculated dose profiles agreed with the measured data within $\pm 0.5 \%$ and $\pm 1.5 \%$ inside the PTV region for the non-IMRT and IMRT tomotherapy deliveries, respectively. Various potential solutions to minimize the effect of target motion were also discussed, and each proposed method needs to be investigated for its effectiveness in suppressing the three dose discrepancies described here. The computer simulation model could also prove useful during the patient selection process for the different approaches of minimizing dose artifacts. 


\section{Cancer Therapy \& Oncology International Journal}

\section{Funding}

This work was supported by project research (81671771) from the Natural Science foundation China.

\section{References}

1. Lu W (2008) Real-time motion-adaptive delivery (MAD) using binary MLC: II. Rotational beam (tomotherapy)delivery. Phys Med Biol 53(22): 6491-511.

2. Hanley J Debois MM, Mah D (1999) Deep inspiration breath-hold technique for lung tumours: the potential value of target immobilization and reduced lung density in dose escalation. Int J Radiat Oncol Biol Phys 45(3): 603-11.

3. Wong JW, Sharpe MB, Jaffray DA, Kini VR, Robertson JM, et al. (1999) The use of active breathing control $(\mathrm{ABC})$ to reduce margin for breathing motion. Int J Radiat Oncol Biol Phys 44(4): 911-9.

4. Mackie TR, Holmes T, Swerdloff S et al. (1993) Tomotherapy: A new concept for the delivery of dynamic conformal radiotherapy. Med Phys 20(6): 1709-1719.

5. Olivera GH, Shepard DM, Ruchala K et al. (1999) "Tomotherapy." In: Van Dyk J ed. The Modern Technology of Radiation Oncology: A Compendium for Medical Physicists and Radiation Oncologists. Madison, WI: Medical Physics Publishing 521-587.

6. Balog J, Mackie TR, Pearson D, Hui S, Paliwal B, et al. (2003) Benchmarking beam alignment for a clinical helical tomotherapy device. Med Phys 30(6): 1118-1127.

7. Kubo HD, Len PM, Minohara S, and Mostafavi H (2000) Breathingsynchronized radiotherapy program at the University of California Davis Cancer Center. Med Phys 27(2): 346-53.

8. Barnes EA, Murray BR, Robinson DM, Underwood LJ, Hanson J, et al. (2001) Dosimetric evaluation of lung tumor immobilization using breath hold at deep inspiration. Int J Radiat Oncol Biol Phys 50(4): 1091-8.

9. Hugo GD, Agazaryan N, Solberg TD (2002) An evaluation of gating window size, delivery method, and composite field dosimetry of respiratory-gated IMRT. Med Phys 29(11): 2517-25.

10. Dietrich L, Tucking T, Nill S, and Oelfke U (2005) Compensation for respiratory motion by gated radiotherapy: an experimental study. Phys Med Biol 50(10): 2405-14.

11. Kapatoes JM, Olivera GH, Schloesser EA, et al. (2001) Gating-byrotation: a solution to the problem of intratreatment motion in helical tomotherapy. Int J Radiat Oncol Biol Phys 51 (suppl 1), 95.

12. Zhang T, Jeraj R, Keller H, Lu W, Olivera GH, et al. (2004) Treatment plan optimization incorporating respiratory motion. Med Phys 31(6): 1576-86.

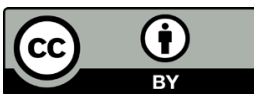

This work is licensed under Creative

Commons Attribution 4.0 License

DOI: 10.19080/CTOIJ.2019.13.555864
13. Kim B, Kron T, Battista JJ, Van Dyk J (2005) Investigation of dose homogeneity for loose helical tomotherapy delivery in the context of breath-hold radiation therapy. Phys Med Biol 50(10): 2387-404.

14. Langen KM and Jones DT (2001) Organ motion and its management. Int J Radiat Oncol Biol Phys 50(1): 265-78.

15. Seppenwoolde Y, Shirato H, Kitamura K, Shimizu S, van Herk M, et al (2002) Precise and real-time measurement of 3D tumor motion in lung due to breathing and heartbeat, measured during radiotherapy. Int J Radiat Oncol Biol Phys 53(4): 822-34.

16. Mageras GS, Pevsner A, Yorke ED, et al. (2004) Measurement of lung tumor motion using respiration-correlated CT. Int J Radiat Oncol Biol Phys 60(3): 933-41.

17. Keall PJ, Mageras GS, Balter JM, et al. (2006) The management of respiratory motion in radiation oncology: report of AAPM Task Group 76. Med Phys 33: 3874-3900.

18. Yang JN, Mackie TR, Reckwerdt P, Deasy JO, Thomadsen BR (1997) An investigation of tomotherapy beam delivery. Med Phys 24(3): 425-36.

19. Yu CX, Jaffray DA, and Wong JW (1998) The effects of intra-fraction organ motion on the delivery of dynamic intensity modulation. Phys Med Biol 43(1): 91-104.

20. Kissick MW, Boswell SA, Jeraj R, Mackie TR (2005) Confirmation, refinement, and extension of a study in intrafraction motion interplay with sliding jaw motion. Med Phys 32(7): 2346-50.

21. Kanagaki B, Read PW, Molloy JA, Larner JM, Sheng K (2007) A motion phantom study on helical tomotherapy: the dosimetric impacts of delivery technique and motion. Phys Med Biol 52(1): 243-55.

22. Theraplan Plus v.3.0 (1998) Technical Reference Manual (Kanata, Canada: MDS Nordion). USA.

23.Zhang T, Lu W, Olivera GH, et al. (2007) Breathing-synchronized delivery: a potential four-dimensional tomotherapy treatment technique Int J Radiat Oncol Biol Physics 68(5): 1572-1578.

24. Kim B, Kron T, Chen J, and Battista J (2006) Preliminary Investigation of Multi-Pass Respiratory Gated Helical Tomotherapy (MRG-HT). AAPM 48th Annual Meeting July 30-August 3, Orlando, FL Med Phys 33, 2285.

25. Kron T, Grigorov G, Yu E et al. (2004) Planning evaluation of radiotherapy for complex lung cancer cases using helical tomotherapy. Phys Med Biol 49(16): 3675-90.

26. Kissick MW, Fenwick J, James JA, et al. (2005) The helical tomotherapy thread effect. Med Phys 32(5): 1414-1423.

27. Johns HE, and Cunningham JR (1983) The Physics of Radiology, (4 $4^{\text {th }}$ edn), Springfield, IL: Thomas, USA.

Your next submission with Juniper Publishers will reach you the below assets

- Quality Editorial service

- Swift Peer Review

- Reprints availability

- E-prints Service

- Manuscript Podcast for convenient understanding

- Global attainment for your research

- Manuscript accessibility in different formats

( Pdf, E-pub, Full Text, Audio)

- Unceasing customer service

Track the below URL for one-step submission

https://juniperpublishers.com/online-submission.php 